\title{
High precision estimation of modeled aerosol direct radiative forcing
}

Fei Luo and Adam Monahan

School of Earth and Ocean Sciences, University of Victoria, Victoria,British Columbia,Canada

\author{
Knut von Salzen*
}

5

Canadian Centre for Climate Modelling and Analysis (CCCma),Environment

Canada, Victoria,British Columbia, Canada

${ }_{7}^{*}$ Corresponding author address: Knut von Salzen, Canadian Centre for Climate Modelling and 8 Analysis (CCCma),Environment Canada,University of Victoria, PO Box 1700, STN CSC, Victo9 ria, BC V8W 2Y2, Canada.

10 E-mail: knut.vonsalzen@ec.gc.ca 


\begin{abstract}
${ }_{11}$ Aerosol radiative forcing can be difficult to quantify both accurately and 12 precisely in global climate models. Long climate model integrations are often 13 required and levels of statistical uncertainty can be substantial for some of the 14 diagnostic methods and diagnosed forcings. Instantaneous estimates of sul15 fate aerosol direct radiative forcings in the present-day climate are compared ${ }_{16}$ to forcings that were determined using different diagnostic methods based on 17 nudged climate model simulations with specified sea surface temperatures and ${ }_{18}$ sea ice. Our results show that the accuracy and precision of aerosol radiative 19 forcing estimates vary considerably among different methods. Nudging of model trajectories to constrain natural meteorological variability in the simu-

${ }_{21}$ lations leads to highly accurate and precise estimates of sulfate radiative forc${ }_{22}$ ings for a wide range of nudging parameters. The radiative forcing of black 23 carbon aerosols, for which instantaneous estimates of the direct radiative forc${ }_{24}$ ing are not available, was also assessed using these methods. We show that 25 the nudging process also significantly increases the precision of these radia${ }_{26}$ tive forcing estimates.
\end{abstract}




\section{Introduction}

Aerosols can cause negative or positive radiative forcings by scattering and absorption of radiation (the direct effect). Absorbing aerosols can also lead to the evaporation of cloud droplets (the semi-direct effect). Furthermore, aerosols can act as Cloud Condensation Nuclei (CCN) and thereby cause changes in cloud properties and associated radiative fluxes (indirect effects), including changes in precipitation efficiencies and cloud lifetimes (the second indirect or cloud lifetime effect).

Uncertainties in the radiative effects of aerosols on climate represent a leading cause of overall uncertainty in climate projections (Boucher et al. 2013).These uncertainties are largely associated with limitations in simulations of aerosol, cloud microphysical, and optical properties in Global Climate Models (GCM). Limited observations of aerosol distributions and radiative properties means that there are currently no practical alternatives to GCM-based estimates of global radiative forcings.

Different methods have been used to diagnose radiative forcing (RF) in GCMs. For instance, instantaneous RFs can be diagnosed as the radiative flux perturbation after an climate forcing agent is introduced, usually by performing additional radiation calculations in the GCM with fixed meteorological conditions. However, this method cannot be used if RFs are intimately tied to changes in clouds, water vapor, and temperature. This is particularly important for aerosol semi-direct and second indirect effects. RF methods that account for dynamic interactions of atmospheric processes are needed to account for these changes. For example, RF can be determined as the difference in radiative fluxes between two simulations, with and without the radiative effects of the original forcing agent included. Such approaches are based on the assumption that meteorological variables respond rapidly to the introduction of a RF agent so that impacts of RFs on sea surface 
temperatures (SSTs) and sea ice (e.g. Lohmann et al. 2010; Boucher et al. 2013) or surface air temperatures (Hansen et al. 2005) can be neglected in order to separate RFs from climate feedback processes.

As simulated radiative fluxes vary in time and space due to model internal variability, the diagnosis of statistically meaningful aerosol radiative forcings often requires multi-decade long simulations or an ensemble of shorter simulations. This fact is particularly important for forcing agents that produce radiative forcings which are much smaller than cloud and snow radiative effects in the simulations, which can easily mask the radiative forcings. However, much shorter model integrations are frequently used in practice, especially for models with costly treatments of complex aerosol and chemical processes. This raises concerns about the precision of these forcing estimates, with potential implications for the accuracy of climate assessments that are based on these estimates.

Kooperman et al. (2012) introduced a method in which RFs were determined as the difference in radiative fluxes between two simulations, with nudging of atmospheric variables in the simulations so that the natural variability in the two simulations was similar. This nudging considerably improved the statistical significance of forcing estimates for aerosol indirect effects. However, they did not use their method to diagnose aerosol direct radiative effects and only used a single model with a very specific implementation of the nudging approach. Furthermore, it is possible that the unphysical nudging of the simulations could improve the precision of the RF estimates while reducing their accuracy. While it is promising, further analysis of the method is needed.

Since uncertainties in diagnostic methods may contribute to overall uncertainties in radiative forcings, it is useful to both quantify and reduce these uncertainties. In the following, the accuracy and precision of some commonly used diagnostic methods for aerosol radiative forcings are evaluated. Two climatologically important types of aerosols with very different radiative effects, 
74

sulfate and black carbon, are considered. The method by Kooperman et al. (2012) is analyzed in detail to determine the influence of nudging variables and parameters on diagnosed forcings.

\section{Methods}

\section{a. Model Summary}

The Canadian Atmospheric Global Climate Model (CanAM4.1) is used to diagnose radiative forcings of sulfate and black carbon aerosols. The model is similar to the earlier version CanAM4 (Von Salzen et al. 2013). The main model improvements that are relevant to this study are a higher vertical resolution in the upper troposphere, a reduced solar constant $\left(1361 \mathrm{Wm}^{-2}\right)$ and an improved treatment of radiative transfer for the solar continuum. The linear grid in the model has a resolution of 128 (longitude) by 64 (latitude) for a spectral resolution of T63 and 49 vertical levels. As described by von Salzen et al. (2013), the model contains a prognostic treatment of bulk aerosols. In this study, present-day aerosol and aerosol precursor emissions from the ECLIPSE emission inventory, version 4a (Klimont et al., in preparation; http://nilu.eclipse.no, and will be also available from http://eccad.sedoo.fr ) are used. Simulations were performed following the specifications outlined by Taylor et al. (2012) for the Atmospheric Model Inter-comparison Project (AMIP), which is a subset of the Coupled Model Inter-comparison Project Phase 5 (CMIP5).

\section{b. Diagnostic Methods for Aerosol Radiative Forcings}

Aerosol direct RFs are diagnosed in this study using different methods, as we will now discuss. For sulfate aerosol, the results are evaluated through comparisons with instantaneous RFs at the top of the atmosphere (TOA), which is diagnosed as the difference in net TAO radiative flux with and without extinction of radiation by aerosols in the model. We also refer to as the true forcing 
because it is a purely diagnostic method which directly samples radiative transfer calculations in the model.

The Effective Radiative Forcing (ERF) (Boucher et al. 2013), also known as radiative flux perturbation (Lohmann et al. 2010; Haywood et al. 2009) or quasi-forcing(Rotstayn and Penner 2001 ) computes the aerosol RF as a radiative flux $(F)$ perturbation at the TOA,

$$
\mathrm{ERF}=F(\text { aerosol })-F(\text { noaerosol }),
$$

based on a pair of simulations with identical SSTs and sea ice conditions. In Eqn (1), $F$ (aerosol) and $F$ (noaerosol) refer respectively to the TOA radiative fluxes for simulations with and without the effect of the aerosol on clear sky radiative transfer. The latter simulation is carried out having set aerosol concentrations to zero in the radiation scheme, which eliminates direct radiative effects from the model but has no direct impacts on other climate forcings and processes.

For simulations with fixed SST but evolving land surface temperatures,Hansen et al. (2005) proposed the following radiative forcing estimate

$$
\text { HansenRF }=\mathrm{ERF}+\delta T_{s} / \lambda,
$$

where $\delta T_{S}$ is the global surface air temperature change and $\lambda$ is the equilibrium climate sensitivity. Here, we use $\lambda=0.96 \mathrm{~K} /\left(\mathrm{Wm}^{-2}\right)$ (Andrews et al. 2012) based on coupled simulations with CanESM2 for doubling of $\mathrm{CO}_{2}$. Hansen RF arguably provides a better estimate of the RF than ERF because it accounts for surface temperature feedbacks. Surface temperature changes result in global-scale temperature changes, so the Hansen RF can only be meaningfully defined on a global average and spatial patterns are not available.

As a result of the chaotic nature of the climate system, the realizations of natural variability will be different in the individual simulations used to determine ERF or Hansen RF. Radiative forcing 
estimates are therefore based on relatively small differences in the means of highly variable quantities. As Kooperman et al. (2012) showed, the impact of natural variability in these simulations can be minimized by nudging simulated horizontal wind and dry static energy to follow a prescribed atmospheric realization simulated by a previous control simulation with the model. Denoting the model state as $X$, the model dynamics and physics $M(X)$ is modified with a nudging term

$$
\frac{\partial X_{m}}{\partial t}=M\left(X_{m}\right)-\frac{X_{m}-X_{p}}{\tau} .
$$

The subscripts $m$ and $p$ denote the nudged and prescribed model states, respectively, and $\tau$ is the relaxation time constant. In general, the nudging is only applied to a subset of model fields. In the current study, different model fields and time scales are used to examine the sensitivity of the method to these choices. In the following, we will refer to nudged effective radiative forcing (NERF) and Hansen nudged radiative forcing (Hansen NERF).

In addition to sulfate, the methods described above were also applied to diagnose RFs for black carbon. However, ERF methods do not filter out responses of clouds to changes in atmospheric heating rates so black carbon RF estimates also include semi-direct effects. Consequently, instantaneous direct radiative forcing estimates cannot be used to evaluate black carbon RFs.

\section{c. Model Simulations}

A 20-member ensemble of simulations was generated to provide control simulations for the RF diagnostic methods. Each control simulation includes sulfate and black carbon radiative effects and started on 1 January 2006, with a 1 year spin-up. The analysis period ran from 1 January 2007 to 31 December 2010, yielding a total of 80 simulated years for the analysis of radiative forcings. In order to determine the direct ERF and corresponding Hansen RF , two further 20- 
member model ensembles were generated without contributions of sulfate aerosol or black carbon to radiative transfer, respectively.

In order to compute the RFs for the nudged simulations, several different 6-member model ensembles were generated. In the first ensemble, the horizontal winds and temperatures were nudged toward the corresponding fields from the first 6 control ensemble members on a timescale of 24 hours. A second model ensemble was generated for a nudging time scale of 6 hours and a third ensemble for the same relaxation time scale but with additional nudging of specific humidity. The sulfate instantaneous RF was determined directly from the radiation code of the control simulations.

\section{Analysis of Diagnosed Radiative Forcings}

Ensemble means and standard deviations of the global-mean sulfate and black carbon RFs are summarized in Table 1. Figure 1 shows the corresponding RFs for individual ensemble members. For sulfate RF, the Hansen RF produces slightly better agreement with the true RF than the ERFs. However, benefits of surface air temperature corrections are relatively small for NERFs owing to highly constrained air temperatures in the nudged simulations. Nudging of model meteorological fields clearly leads to a much smaller spread among ensemble members and therefore higher precision, especially for the most strongly constrained approach with nudging of winds, temperatures, and specific humidity. An important result of these calculations is that the higher precision of the estimates does not come at the expense of their accuracy (Table 1).

To assess differences in ensemble-mean RF estimates between different methods, Table 2 summarizes P-values of student's T-tests for both sulfate and black carbon. Before T-tests are carried out, the F-test for equal variance between two samples was conducted and the appropriate T-test used. For a P-value that is lower than 0.05 , it can be inferred that differences in mean RF exist 
at a 95\% confidence level. ERF and NERF estimates are almost all statistically distinguishable with the exception of the sulfate Hansen RF, RF estimates are indistinguishable between NERF estimates, which indicates that details of the nudging approach concerning the choice of relaxation time scale or variables are not critical for overall results of this method.

Nudging leads to a slightly higher true RF than in the free control run (Table 1), which can only be explained by small changes in simulated climate with this approach. While differences are significant (Table 2), they are very small.

P-values among individual RF diagnostic methods are summarized in Table 3 . Results in Table 3 confirm that the Hansen method improves the agreement between the ERF and true RF for sulfate. Interestingly, the most strongly constrained nudging approach producing the highest precision (D), results in a RF that is significantly different from the true forcing, even with the Hansen correction of the RF. Owing to the very high precision of the method, relatively small differences in the mean RF can be detected by this method, even if differences may not be very meaningful in practical applications of the method.

In addition to global RFs, spatial patterns of RF are of interest for studies of regional climate processes. Figure 2 shows maps of annual mean ERF and NERF for the different methods, both for sulfate and black carbon. The sulfate RF is strongly negative in coastal areas of China, which can be explained by strong fossil fuel sources of sulfate in this region, while volcanic emissions contribute to the RF over the Mediterranean. With nudging, RF fields agree much better with the true RF, even though the number of ensemble members is smaller than for the ERF (6 vs. 20).

Results for black carbon are broadly consistent with results for sulfate. The black carbon RF is particularly variable in the Pacific and Atlantic subtropic Ocean regions, which is considerably reduced with nudging. 


\section{This paper is a non-peer reviewed preprint submitted to EarthArXiv.}

Differences in sulfate spatial RF patterns are summarized in a Taylor diagram (Taylor 2001)

(Figure 3). Estimates of NERF agree better with the true forcing than the ERF, especially for simulations with short nudging time scale and nudging of moisture.

\section{Summary}

Effective Radiative Forcing (ERF) estimates of the global sulfate direct radiative forcing without properly accounting for impacts of RFs on surface air temperatures in diagnostic calculations are significantly different from instantaneous RF estimates for present-day climate from simulations with CanAM4.1. The precision of estimates of aerosol radiative forcing based on differences between model simulations with and without aerosols is generally limited by model internal variability. The spatial patterns of Effective Radiative Forcing for sulfate and black carbon aerosols diagnosed in CanAM4.1 display clearly unphysical features even after averaging over 80 years of simulation. A considerably improved method for diagnosing sulfate RFs is obtained by constraining model fields by nudging which reduces the difference in internal variability between simulations with and without aerosol radiative forcing. Although the nudging slightly changes the simulated climate and causes statistically significant biases in forcing estimates, the overall accuracy and precision of the method is very high compared to unconstrained RF estimates, even with a much lower number of ensemble members. Variations in details of the nudging approach (relaxation time and nudged fields) causes differences that are very small compared to overall improvements in RF estimates. Furthermore, the method does not require an additional correction of diagnosed RFs to account for changes in surface air temperatures, in marked contrast to unconstrained ERF estimates. Similar benefits of nudging are also found for the diagnosis of black carbon radiative effects. 
Overall, the nudged ERF method can be recommended for the diagnosis of aerosol direct RFs in GCMs.

\section{Acknowledgements}

The authors wish to thank Jason Cole for helpful comments.

\section{References}

Andrews, T., J. M. Gregory, M. J. Webb, and K. E. Taylor, 2012: Forcing, feedbacks and climate sensitivity in CMIP5 coupled atmosphere-ocean climate models. Geophysical Research Letters, 39 (9).

Boucher, O., et al., 2013: Clouds and aerosols. in: Climate change 2013: The physical science basis. Contribution of Working Group I to the Fifth Assessment Report of the Intergovernmental Panel on Climate Change [Stocker, T.F., D. Qin, G.-K. Plattner, M. Tignor, S.K. Allen, J. Boschung, A. Nauels, Y. Xia, V. Bex and P.M. Midgley (eds.)].

Hansen, J., et al., 2005: Efficacy of climate forcings. Journal of Geophysical Research: Atmospheres (1984-2012), 110 (D18).

Haywood, J., L. Donner, A. Jones, and J. Golaz, 2009: Global indirect radiative forcing caused by aerosols: IPCC (2007) and beyond. MIT Press, 451-467 pp.

Kooperman, G. J., M. S. Pritchard, S. J. Ghan, M. Wang, R. C. Somerville, and L. M. Russell, 2012: Constraining the influence of natural variability to improve estimates of global aerosol indirect effects in a nudged version of the Community Atmosphere Model 5. Journal of Geophysical Research: Atmospheres (1984-2012), 117 (D23). 
Lohmann, U., et al., 2010: Total aerosol effect: radiative forcing or radiative flux perturbation? Atmospheric Chemistry and Physics, 10 (7), 3235-3246.

Rotstayn, L. D. and J. E. Penner, 2001: Indirect aerosol forcing, quasi forcing, and climate response. Journal of climate, 14 (13), 2960-2975.

Taylor, K. E., 2001: Summarizing multiple aspects of model performance in a single diagram. Journal of Geophysical Research: Atmospheres (1984-2012), 106 (D7), 7183-7192.

Taylor, K. E., R. J. Stouffer, and G. A. Meehl, 2012: An overview of CMIP5 and the experiment design. Bulletin of the American Meteorological Society, 93 (4), 485-498.

von Salzen, K., et al., 2013: The Canadian fourth generation atmospheric global climate model (CanAM4). Part I: representation of physical processes. Atmosphere-Ocean, 51 (1), 104-125. 


\section{LIST OF TABLES}

Table 1. Estimated global-mean radiative forcing for different diagnostic methods. A: ERF. B: NERF, with 24-hour relaxation time scale. C: NERF, with 6-hour relaxation time scale. D: NERF, Additional nudging of specific humidity. Within each row, the upper set of numbers are for sulfate aerosol and the lower row for black carbon.

Table 2. P values of T-tests of differences between global mean radiative forcing as estimated by the different methods.

Table 3. As in table 2, for tests of differences between true RF, ERF, and Hansen RF estimates for individual methods. 
TABLE 1. Estimated global-mean radiative forcing for different diagnostic methods. A: ERF. B: NERF, with 24-hour relaxation time scale. C: NERF, with 6-hour relaxation time scale. D: NERF, Additional nudging of specific humidity. Within each row, the upper set of numbers are for sulfate aerosol and the lower row for black carbon.

\begin{tabular}{cccc}
\hline \hline & True Forcing $\left(\mathrm{W} \mathrm{m}^{-2}\right)$ & $\mathrm{RF}\left(\mathrm{W} \mathrm{m}^{-2}\right)$ & Hansen RF $\left(\mathrm{W} \mathrm{m}^{-2}\right)$ \\
\hline $\mathrm{A}$ & $-0.6022 \pm 0.0052$ & $-0.5428 \pm 0.1082$ & $-0.5700 \pm 0.1037$ \\
& & $0.3372 \pm 0.1273$ & $0.3559 \pm 0.1334$ \\
$\mathrm{~B}$ & $-0.6076 \pm 0.0055$ & $-0.5970 \pm 0.0123$ & $-0.6073 \pm 0.0128$ \\
& & $0.4508 \pm 0.0240$ & $0.4457 \pm 0.0229$ \\
$\mathrm{C}$ & $-0.6136 \pm 0.0044$ & $-0.6002 \pm 0.0205$ & $-0.6073 \pm 0.0211$ \\
& & $0.4390 \pm 0.0182$ & $0.4326 \pm 0.0180$ \\
& & $-0.5881 \pm 0.0060$ & $-0.5957 \pm 0.0059$ \\
$\mathrm{D}$ & $-0.6102 \pm 0.0054$ & $0.4347 \pm 0.0099$ & $0.4281 \pm 0.0100$ \\
\hline
\end{tabular}


TABLE 2. P values of T-tests of differences between global mean radiative forcing as estimated by the different methods.

\begin{tabular}{cccccccc}
\hline \hline \multirow{2}{*}{ Sulfate } & & A vs. B & A vs. C & A vs. D & B vs. C & B vs. D & C vs. D \\
\hline \multirow{5}{*}{ True RF } & $\mathbf{0 . 0 4 0}$ & $\mathbf{6 . 7} \times \mathbf{1 0}^{-\mathbf{5}}$ & $\mathbf{3 . 3} \times \mathbf{1 0}^{-\mathbf{3}}$ & 0.063 & 0.42 & 0.26 \\
& ERF & $\mathbf{0 . 0 4 0}$ & $\mathbf{0 . 0 3 5}$ & 0.078 & 0.75 & 0.14 & 0.21 \\
& Hansen RF & 0.13 & 0.14 & 0.28 & 0.99 & 0.072 & 0.24 \\
& ERF & $\mathbf{1 . 0} \times \mathbf{1 0}^{-\mathbf{3}}$ & $\mathbf{2 . 3} \times \mathbf{1 0}^{-\mathbf{3}}$ & $\mathbf{2 . 9} \times \mathbf{1 0}^{-\mathbf{3}}$ & 0.36 & 0.16 & 0.62 \\
& Hansen RF & $\mathbf{8 . 8} \times \mathbf{1 0}^{-\mathbf{3}}$ & $\mathbf{0 . 0 2 1}$ & $\mathbf{0 . 0 2 6}$ & 0.30 & 0.12 & 0.60 \\
\hline
\end{tabular}


TABLE 3. As in table 2, for tests of differences between true RF, ERF, and Hansen RF estimates for individual methods.

\begin{tabular}{cccccccc}
\hline \hline \multirow{2}{*}{ Sulfate } & & & A & B & C & D \\
& True vs. ERF & $\mathbf{0 . 0 2 4}$ & 0.082 & 0.17 & $\mathbf{5 . 0} \times \mathbf{1 0}^{-\mathbf{5}}$ \\
& True vs. & Hansen RF & 0.18 & 0.96 & 0.51 & $\mathbf{1 . 2} \times \mathbf{1 0}^{-\mathbf{3}}$ \\
& ERF vs. Hansen RF & 0.42 & 0.19 & 0.57 & 0.052 \\
\multirow{2}{*}{ Black Carbon } & ERF vs. Hansen RF & 0.65 & 0.71 & 0.55 & 0.28 \\
\hline
\end{tabular}




\section{LIST OF FIGURES}

Fig. 1. Global-mean estimates of sulfate and black carbon radiative forcing from both ERF (circle) and Hansen (inverted triangle) methods. Experiments are as described in the caption of

Fig. 2. Maps of ERF sulfate radiative forcing (upper) and black carbon radiative forcing (bottom) for different nudging parameters. In the panel beside each map, the red line is zonal mean of the true forcing for sulfate and the blue line is the ERF estimate in both sulfate and black carbon radiative forcing estimates.

Fig. 3. Taylor diagram of ensemble-mean sulfate radiative forcing for the nudged ERF simulations relative to the true forcing. The different experiments (A through D) are as listed in the caption of Figure 1. 

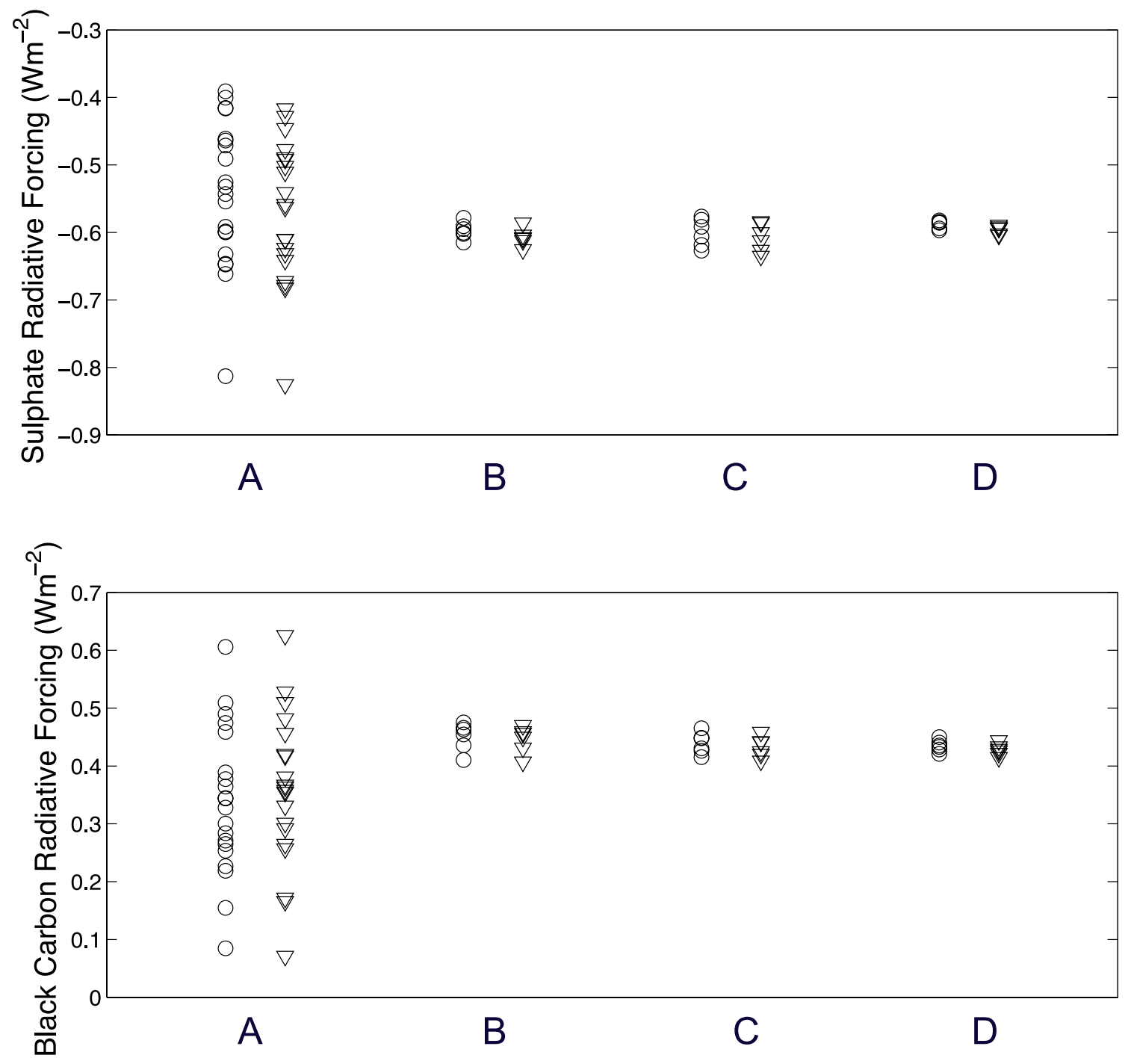

FIG. 1. Global-mean estimates of sulfate and black carbon radiative forcing from both ERF (circle) and Hansen (inverted triangle) methods. Experiments are as described in the caption of Table 1. 

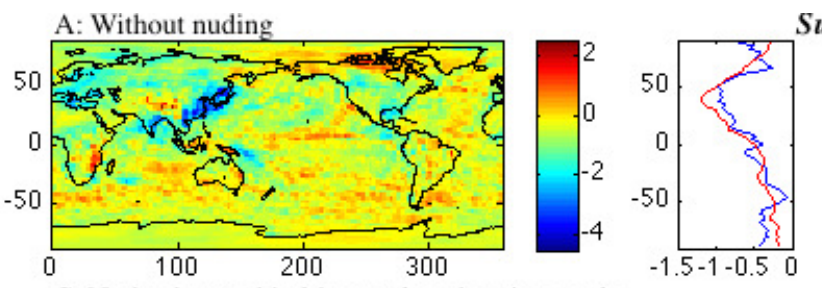

Sulfate B: Nudged run with 24-hour relaxation time scale
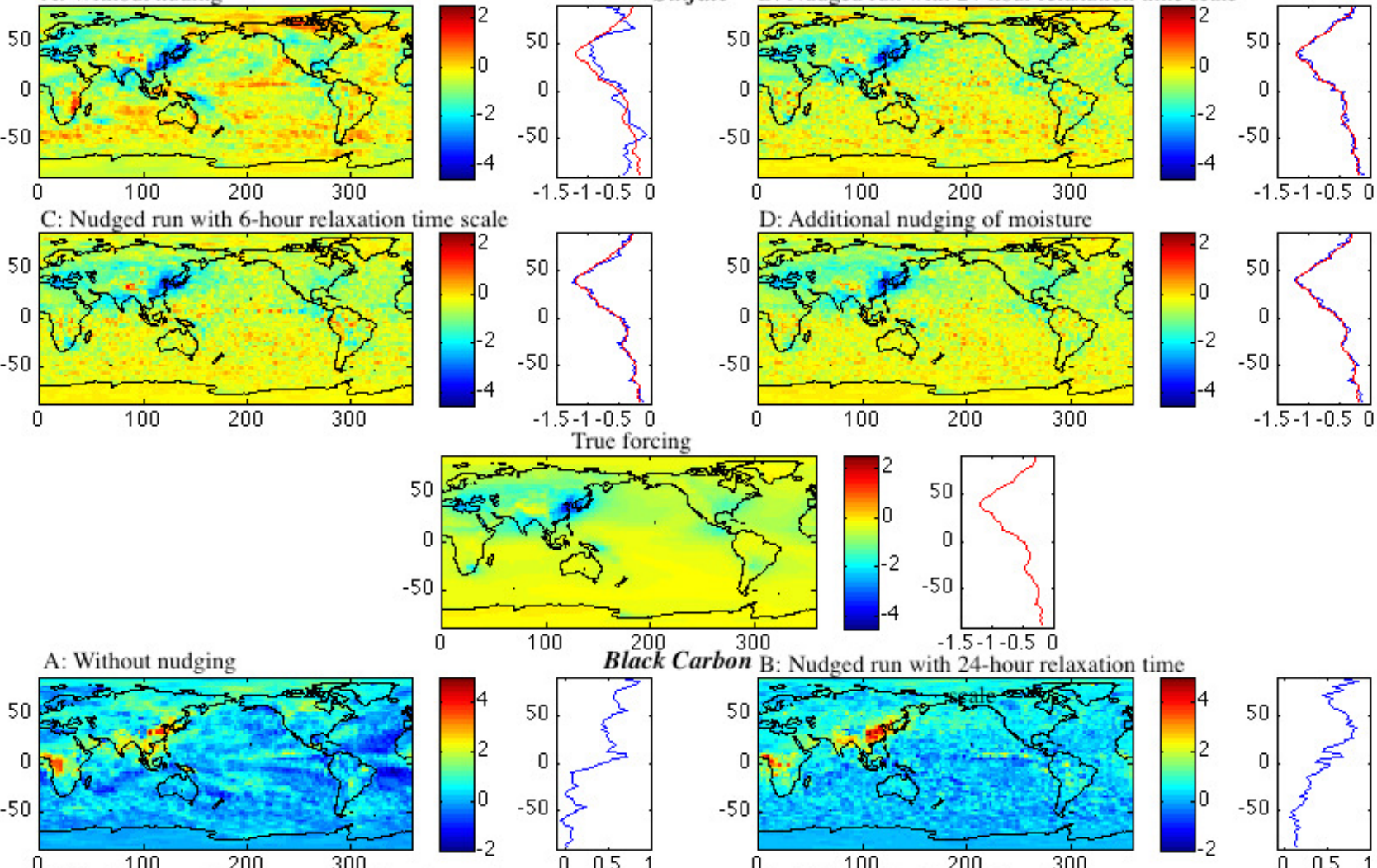

$-1.5-1-0.50$
True forcing
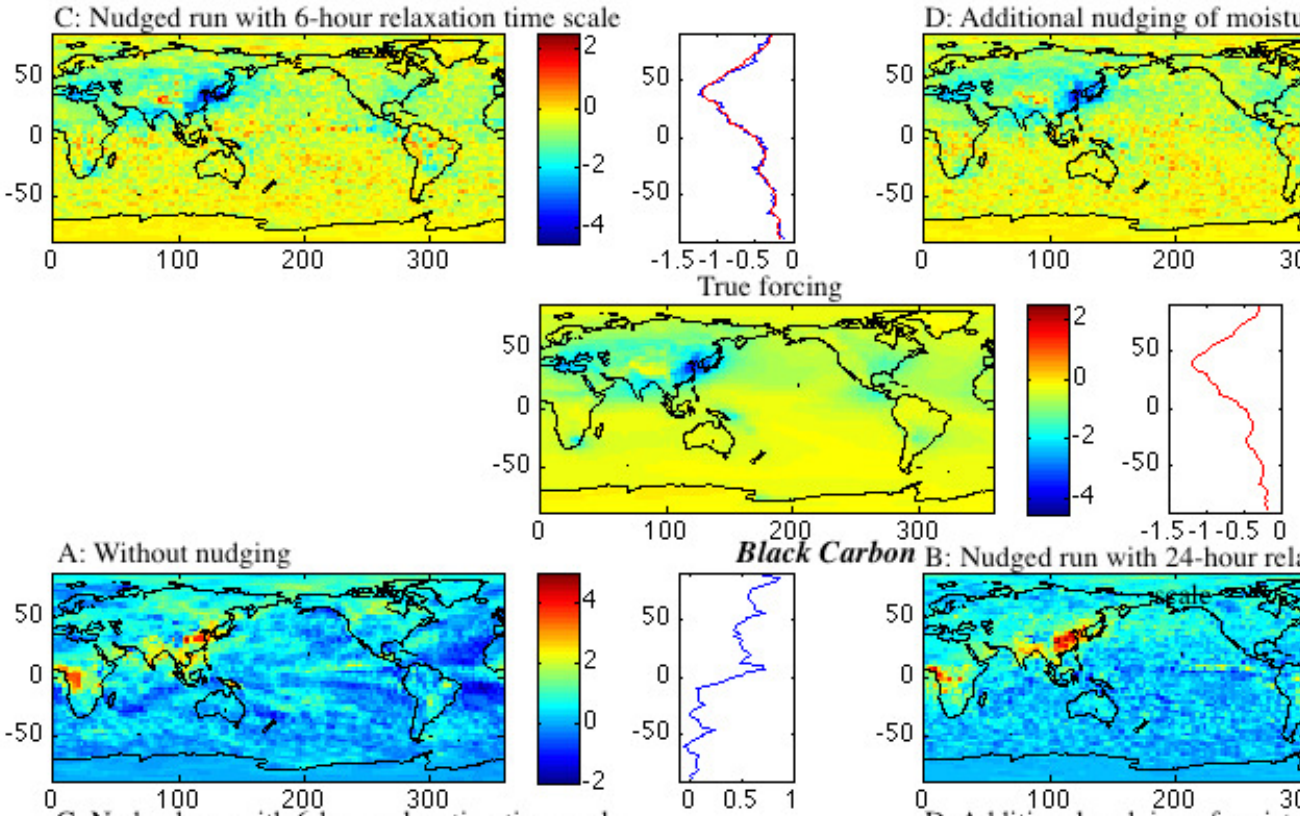

Black Carbon B: Nudged run with 24-hour relaxation time
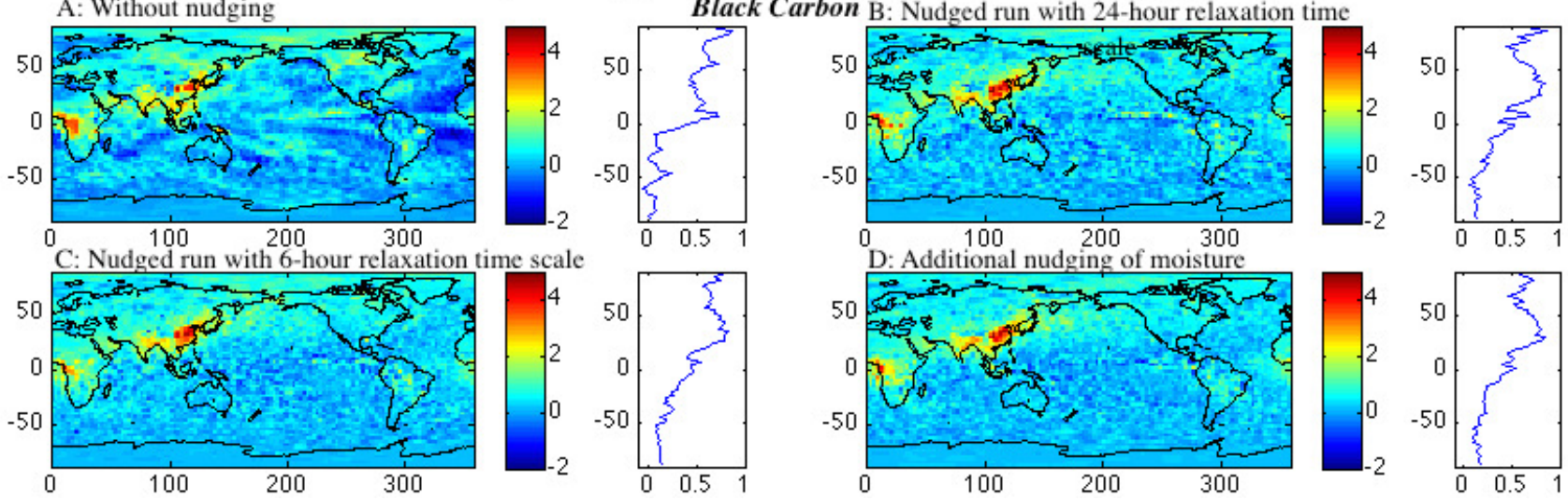

FIG. 2. Maps of ERF sulfate radiative forcing (upper) and black carbon radiative forcing (bottom) for different

nudging parameters. In the panel beside each map, the red line is zonal mean of the true forcing for sulfate and the blue line is the ERF estimate in both sulfate and black carbon radiative forcing estimates. 


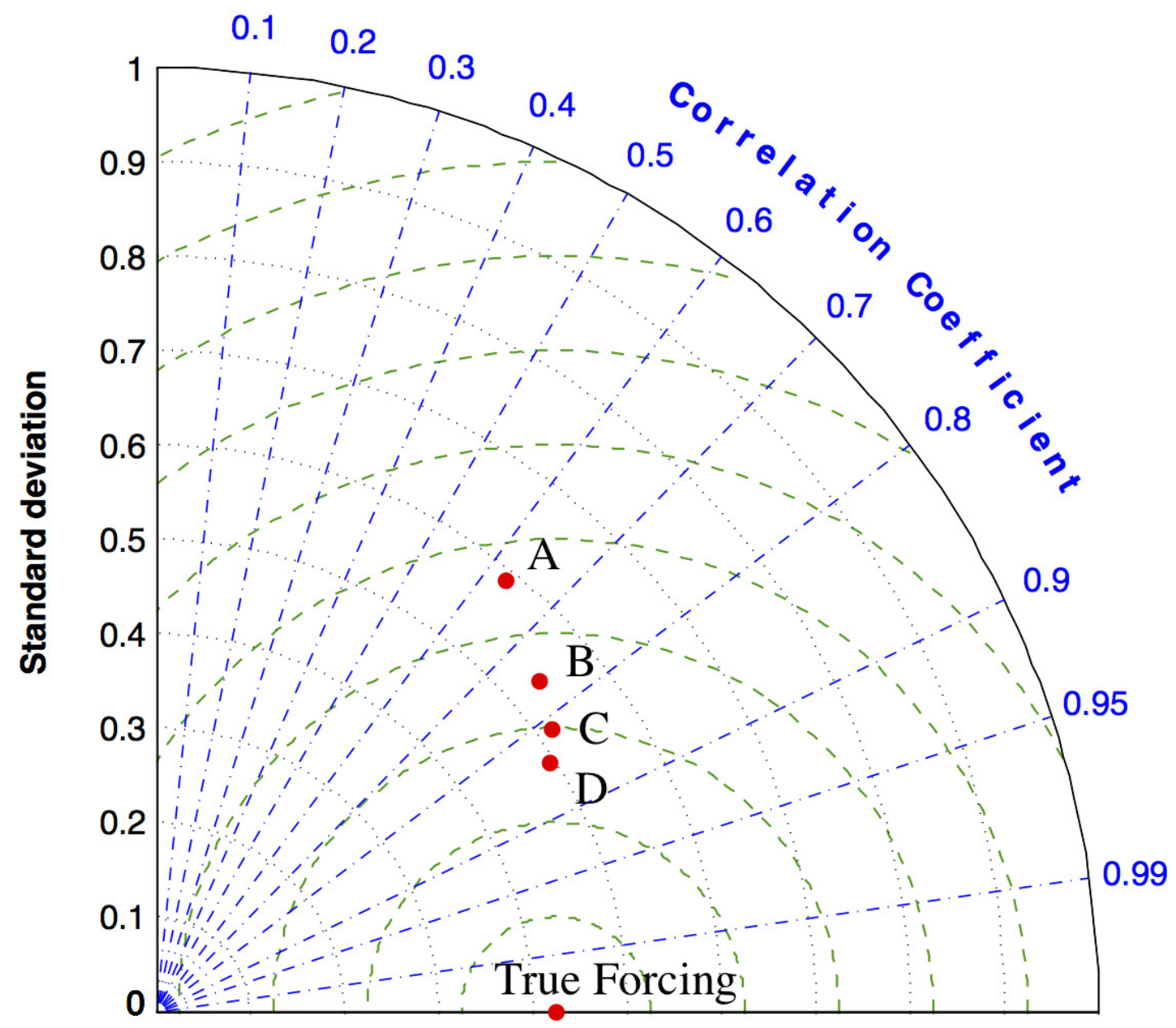

FIG. 3. Taylor diagram of ensemble-mean sulfate radiative forcing for the nudged ERF simulations relative to the true forcing. The different experiments (A through D) are as listed in the caption of Figure 1. 